\title{
Athlete's Heart in Elite Sport Climbers: Cardiac Adaptations Determined Using ECG and Echocardiography Data
}

\author{
Isabelle Schöffl, $\mathrm{PhD}, \mathrm{MD}, \mathrm{MSc}^{1,2,3}$; Jan Wüstenfeld, $\mathrm{MD}^{4}$; Gareth Jones, $\mathrm{PhD}, \mathrm{MSc}^{3}$; Sven Dittrich, $\mathrm{PhD} \mathrm{MD}^{1}$; \\ Chris Lutter, MD, MHBA ${ }^{5}$; Volker Schöffl, PhD, MD, MHBA ${ }^{2,3,6}$
}

${ }^{1}$ Department of Pediatric Cardiology, University Hospital Erlangen-Nuremberg, Erlangen, Germany; ${ }^{2}$ Section of Sportsmedicine and Sports Orthopaedics, Department of Orthopedic and Trauma Surgery, Klinikum Bamberg, Bamberg, Germany; ${ }^{3}$ School of Clinical and Applied Sciences, Leeds Beckett University, Leeds, United Kingdom; ${ }^{4}$ Insitute for Applied Exercise Science, University Leipzig, Leipzig, Germany; ${ }^{5}$ Department of Orthopaedics, University Hospital Rostock, Rostock, Germany; ${ }^{6}$ Department of Emergency Medicine, Section Wilderness Medicine, University of Colorado School of Medicine, Denver, $C O$

Introduction - Sudden cardiac death in a young athlete is the leading cause of mortality in athletes during sport. Specific knowledge about cardiac adaptations are necessary for a better understanding of the underlying causes of such events.

Methods-A retrospective analysis of the electrocardiogram and echocardiographic data obtained during the yearly medical examination of the entire German junior national climbing team was undertaken. First, data from 1 examination were used. In a second step, data from 2 examinations spaced 2 y apart were analyzed for a selected subgroup to gain more knowledge about adaptations to climbing. The data from the subgroup were compared to an age- and sex-matched control group of Nordic skiers from the German junior national Nordic skiing team.

Results-Forty-seven young climbers (20 girls, 27 boys) were examined once. There were no pathological findings in the electrocardiogram or echocardiography. The left ventricular (LV) measurements fell between those for athletes and nonathletes. Eight boys and 6 girls from this group were tested twice over a timeframe of $27.5 \mathrm{mo}$. All LV measurements increased over time. After $2 \mathrm{y}$, the measurements from the climbers were comparable to those of the Nordic skiers.

Conclusions - Hypertrophic cardiomyopathy (hypertrophy of the LV) is the leading cause of sudden cardiac death in athletes. An increase in LV dimensions was observed in the young climbers in this study. LV dimensions being comparable to high-level Nordic skiers after $2 \mathrm{y}$ in the national team imply structural changes over time in this cohort.

Keywords: pediatric cardiology, adolescent athlete, cardiomyopathy, sports ability test, eccentric hypertrophy, concentric hypertrophy

\section{Introduction}

The term athlete's heart syndrome refers to the electrophysiological, structural, and functional myocardial adaptations associated with continuous training stimuli in the absence of pathologic significance. ${ }^{1}$ Such

Corresponding author: Isabelle Schöffl, $\mathrm{PhD}, \mathrm{MD}, \mathrm{MSc}$, Department of Pediatric Cardiology, University Hospital ErlangenNuremberg, Loschgestrasse 15, 91054 Erlangen, Germany; e-mail: isabelle.schoeffl@me.com.

Submitted for publication January 2020.

Accepted for publication July 2020. adaptations may include a 10 to $15 \%$ increase in the chamber dimensions of the left and right ventricles and a 10 to $20 \%$ increase in left ventricular wall thickness, resulting in increased stroke volume and improved cardiac filling in diastole. ${ }^{2}$ The effect of exercise on the cardiovascular system largely depends on the underlying stimulus (eg, endurance or strength training). ${ }^{1}$ Endurance-based training effects include reduced systolic blood pressure and increased cardiac output, thus decreasing myocardial oxygen consumption. ${ }^{1}$ Myocardial oxygen consumption is defined as coronary blood flow $\times$ arteriovenous difference in $\mathrm{O}_{2}$ content. In contrast, 
strength-based training raises blood pressure and heart rate with a resultant increase in myocardial oxygen consumption. ${ }^{1}$ Despite the positive benefits to athletic performance from cardiac adaptation, the high training loads undertaken by elite athletes (defined here as athletes at National Team standard) could potentially lead to adverse adaptations resulting in sudden cardiac death (SCD). ${ }^{2}$ Of note, intense physical exercise in an athlete with an underlying cardiovascular abnormality can also lead to SCD, ${ }^{3}$ usually as a result of an exercise-induced catecholamine surge acting on an arrhythmogenic substrate, invoking ventricular arrhythmia. ${ }^{4}$

Atherosclerosis of the coronary artery is the leading cause of SCD $(80 \%)$ in athletes over the age of $35 \mathrm{y}$. In contrast, inherited and acquired cardiovascular abnormalities with hypertrophic cardiomyopathies (HCM) and arrhythmogenic ventricular cardiomyopathy (AVCM) are the predominant diagnoses in younger athletes. ${ }^{5} \mathrm{HCM}$ classically presents with left ventricular hypertrophy (LVH) without adequate loading conditions in the form of increased physical activity. SCD pathology commonly presents as a result of ventricular tachycardia/fibrillation. ${ }^{6}$ The prevalence of $\mathrm{HCM}$ is estimated to be about $0.2 \%$ in the general population and 0.07 to $0.08 \%$ in athletes. ${ }^{7}$ Interestingly, SCD due to HCM is more common in high dynamic and low isometric intensity intermittent activity sports such as soccer and basketball and is rare in endurance-based sports, probably as a consequence of HCM limiting the endurance capacity of athletes and thus self-selecting them out of such sports. ${ }^{8}$ When investigating the characteristics of climbing using cardiopulmonary exercise testing, the maximal oxygen consumption $\dot{\mathrm{V}}{ }_{2 \max }$ was comparable to that in intermittent activity sports. ${ }^{9}$ However, whether this similarity in the endurance capacity of climbers reflects a higher risk for SCD with HCM cannot be concluded from these data.

Other attributable causes of SCD in young athletes are congenital coronary artery anomalies, aortic dissection and stenosis (Marfan syndrome), electrical cardiac anomalies (Wolff-Parkinson-White syndrome, long QT syndrome, Brugada syndrome, catecholaminergic polymorphic ventricular tachycardia, acquired cardiac abnormalities such as myocarditis), and toxicity from drugs. ${ }^{8}$

Overall, the incidence rate of SCD among young athletes is reported to fall between 2.3 and 4.4/100,000 per year, ${ }^{10-12}$ with a male predominance. ${ }^{13}$ The majority of nontraumatic deaths are attributed to cardiovascular abnormalities identifiable by pre-participation cardiovascular screening and effectively managed by lifestyle modifications (abstinence from exercise, pharmacotherapy, implantable cardioverter-defibrillators). ${ }^{8}$ Routine screening of young athletes throughout their careers to detect early pathophysiologic changes and to better develop an understanding of the impact of individual sports in regard to pathophysiologic adaptation is essential.

An electrocardiogram (ECG) is considered the first-line test for the diagnosis of heart disease in athletes. ${ }^{14}$ Adolescent athletes demonstrate a greater prevalence of trainingrelated and training-unrelated ECG changes than nonathletes, such as a longer PR interval, greater frequency of sinus bradycardia, first-degree atrioventricular (AV)block, incomplete right bundle branch block (IRBBB), voltage criteria for LVH, and early repolarization. ${ }^{15}$ Even though its use for detecting athletes at risk of SCD is controversial, ${ }^{16}$ ECG has clinical utility in identifying adaptations such as LVH or detecting dangerous arrhythmias such as Wolff-Parkinson-White and long QT syndrome in young athletes. ${ }^{17}$ Furthermore, a consensus statement has recommended the implementation of a common European screening protocol based on the use of 12lead ECG. ${ }^{18}$

However, some cardiac structural changes can be missed on ECG but can be identified by echocardiography. ${ }^{19} \mathrm{HCM}$ is the leading cause of SCD in young athletes ${ }^{20}$ and initially may present as LVH. ${ }^{6}$ Although LVH in athletes is not as pronounced as in HCM, the differentiation of HCM in athletes can present difficulties because some athletes develop physiologic LVH with measurements close to those in HCM. ${ }^{21-23}$

Key parameters for investigating the adolescent heart using echocardiography and ruling out cardiomyopathy are left ventricular end-diastolic diameter (LVEDD), maximal septal thickness, left ventricular wall thickness, the transmitral E/A ratio, and the dimensions of the left atrium. ${ }^{15,24,25}$ The E/A ratio represents the ratio of early (E) to late (A) ventricular filling velocities. In ventricular hypertrophy, the left ventricular wall becomes stiffer, which slows the early filling velocity and thus lowers the E/A ratio. Coronary artery anomalies and AVCM may be detected by echocardiographic screening, further reducing the risk of SCD in young athletes.

There is a paucity of research focusing on cardiac screening in young climbers. In Germany, each member of the German Junior National Climbing Team (GJNTC) routinely undergoes a mandatory medical examination. To estimate the effects of climbing at an elite level on the left ventricular dimensions, data from $5 \mathrm{y}$ of this medical examination were analyzed retrospectively. Aside from being able to evaluate a relatively large number of high-level climbers in a cross-sectional design, we retrieved longitudinal data for a smaller number of athletes who remained on the team for at least $2 \mathrm{y}$. Because $2 \mathrm{y}$ of regular endurance training is believed to lead to characteristics of the athlete's heart, ${ }^{26}$ this seemed an appropriate time interval. To clearly 
Table 1. Anthropometric data of the athletes according to age and sex (mean \pm SD with Z-scores in parentheses)

\begin{tabular}{lllllll}
\hline $\begin{array}{l}\text { Age } \\
(y)\end{array}$ & Sex & $\begin{array}{l}\text { Subjects } \\
(\mathrm{n})\end{array}$ & $\begin{array}{l}\text { Height } \\
(\mathrm{cm})\end{array}$ & $\begin{array}{l}\text { Weight } \\
(\mathrm{kg})\end{array}$ & $\begin{array}{l}\text { BMI } \\
\left(\mathrm{kg} \cdot \mathrm{m}^{-2}\right)\end{array}$ \\
\hline \multirow{2}{1}{$12-13$} & male & 4 & $163 \pm 7(-0.4)$ & $47.1 \pm 9.5(-0.8)$ & $17.7 \pm 2.2(-0.1)$ & $\begin{array}{l}\text { Body Fat } \\
(\%)\end{array}$ \\
& female & 1 & $166(0.4)$ & $58.2(0.3)$ & $20.9(0.2)$ & 18 \\
$14-15$ & male & 7 & $171 \pm 6(-0.4)$ & $58.9 \pm 6.7(-0.5)$ & $20.1 \pm 1.4(-0.3)$ & $5 \pm 2$ \\
& female & 5 & $163 \pm 3(-0.3)$ & $55.3 \pm 3.5(-0.4)$ & $20.9 \pm 0.6(-0.2)$ & $14 \pm 2$ \\
$16-17$ & male & 16 & $179 \pm 5(-0.2)$ & $66.7 \pm 6.8(-0.2)$ & $20.9 \pm 1.5(-0.2)$ & $6 \pm 8$ \\
& female & 14 & $166 \pm 7(-0.3)$ & $55.6 \pm 6.4(-0.5)$ & $19.7 \pm 1.8(-0.4)$ & $13 \pm 4$ \\
\hline
\end{tabular}

differentiate the adaptations observed in the climbing team, we chose a typical endurance sport as control: athletes from the German Junior National Team of Nordic skiing (GJNTN). The aim of this study was to determine the effects of climbing at an elite level on the athlete's heart at a young age to establish screening methods for detecting risk for SCD. The hypothesis was that climbing at an elite level would lead to structural changes detectable by echocardiography and ECG.

\section{Methods}

All participants (Table 1) and their respective legal guardians gave written, informed consent to participate in this study. The ethics committee of the University of Erlangen-Nürnberg, FRG approved the study.

Routinely collected data over a 5-y period were examined. Every athlete on the GJNTC has been and still is required to undergo a medical examination to be allowed to participate in international competitions. As a convenience sample, no blinding procedures were undertaken. Some athletes were screened multiple times because they were selected for the team several years in a row. In these cases, only the most recent examination was analyzed, the rationale being the greatest cardiovascular adaptations are likely associated with number of years climbing. The athletes who remained on the team for a minimum duration of $2 \mathrm{y}$ were reevaluated after that timeframe, and the datasets of these 2 medical examinations were compared. The goal was to determine the cardiovascular adaptations of climbing at an elite level after a minimum of 24 mo.

We compared the longitudinal data from those athletes with an age-matched control group of Nordic skiers from the GJNTN - a representative endurance sport. Because members of both teams (skiing and climbing) are comparable in height and weight and with respect to age and sex, no blinding or further matching was possible.

A standard ECG was performed at rest, and the PR interval (atrial depolarization), QT interval (ventricular de- and repolarization), and deviations from the standard findings according to age were recorded. Clinically significant abnormal findings were recorded when one of the following anomalies was found: T-wave inversions in unexpected chest leads according to age or ST segment depressions, pathologic Q waves, complete left bundle branch block, epsilon wave (sign of AVCM), ventricular pre-excitation, Brugada Type 1 pattern, profound sinus bradycardia $(<30$ beats $\left.\cdot \min ^{-1}\right)$, AV block, polymorphic ventricular complexes, and atrial or ventricular arrhythmias. The ECG results were interpreted by a cardiologist and an independent pediatric cardiologist. The pediatric cardiologist was an expert in the clinical peculiarities of childhood ECGs. Any disagreements were arbitrated by the chief of staff of the pediatric cardiology department. Because this was part of a routine medical examination, no blinding for the readings of the ECG was involved.

Because the data used in this study were recorded over a timeframe of $5 \mathrm{y}$ and extracted from a regular medical examination of the GJNTC, it was impossible to ensure that all echocardiographic examinations were undertaken by the same cardiologist. As a consequence, the echocardiographic examinations were performed by 2 senior cardiologists.

A protocol for examining athletes from the national team was designed by our cardiologists. At the time of data collection, a standardized protocol was not available and therefore the protocol used may not be comparable to those now used by other research teams. ${ }^{27}$ For the examination, an EPIQ 7C (Philipps, Eindhoven, Netherlands) was used. The following measurements were recorded: LVEDD, interventricular septum thickness during diastole (IVSD), left ventricular posterior wall thickness (LVPW), and E/A ratio at the mitral valve. The dimensions of the left atrium were measured in the apical 4-chamber view using 2dimensional plotting of the left atrium. These measurements were converted into Z-scores for comparison using an online Z-score calculator (http://www.parameterz.com/ refs/lopez-circimaging-2017).

Anatomical variances of abnormal findings were also recorded. These included right ventricular dilation or dysfunction as sign of AVCM, congenital coronary artery anomalies, signs of aortic pathologies such as dissection or 
Table 2. Anthropometric data of the climbers and the Nordic skiers in the longitudinal approach according to sex (mean $\pm \mathrm{SD}$ )

\begin{tabular}{llllll}
\hline & \multicolumn{2}{l}{ Climbers } & \multicolumn{3}{c}{$\begin{array}{l}\text { Cross-country } \\
\text { skiers }\end{array}$} \\
\cline { 2 - 3 } \cline { 5 - 6 } & Male & Female & & Male & Female \\
\hline Age $(\mathrm{y})$ & $15.8 \pm 0.4$ & $16.0 \pm 0.3$ & $15.0 \pm 0.4$ & $15.1 \pm 0.3$ \\
Height $(\mathrm{cm})$ & $177 \pm 2$ & $163 \pm 1$ & $177 \pm 3$ & $163 \pm 1$ \\
Weight $(\mathrm{kg})$ & $62.8 \pm 2.0$ & $51.6 \pm 1.4$ & $61.3 \pm 3.6$ & $50.4 \pm 2.4$ \\
BMI $\left(\mathrm{kg} \cdot \mathrm{m}^{-2}\right)$ & $20.1 \pm 1.2$ & $19.5 \pm 1.3$ & $19.3 \pm 1.5$ & $18.6 \pm 2.2$ \\
\hline
\end{tabular}

rupture, bicuspid aortic valve or other cause of aortic stenosis, mitral valve prolapse, and pulmonary hypertension measured through Doppler analysis of tricuspid insufficiency.

Statistical analysis was performed using Microsoft Excel 2000 for data collection and SPSS 12.0 (SPSS Inc., Chicago, IL). All measured values are reported as mean \pm SD. The Kolmogorov-Smirnov test was used to check for normal distribution. Homogeneity of variance analyzed by Levine's F-test. For approximately normally distributed variables, differences in means between 2 related groups were analyzed using paired (dependent) $t$ tests; for nonnormally distributed data, Wilcoxon and/or Mann-Whitney $U$ tests were used. For normally distributed variables, differences between climbers and cross-Nordic skiers were analyzed using unpaired (independent) $t$ tests; for nonnormally distributed data, Wilcoxon or Mann-Whitney $U$ tests were used. All tests were 2-tailed, and a 5\% level was considered significant.

\section{Results}

A total of 47 adolescent (mean age 15.6 y) climbers were examined in the time frame of the study; 27 were male and 20 were female. Fourteen climbers were reevaluated after $27.5 \pm 5.4$ mo ( 8 boys and 6 girls) and compared to a group of 8 boys and 6 girls from the GJNTN who were evaluated twice within $28.7 \pm 9.8$ mo. Both groups were comparable with respect to age, height, and weight (Table 2).

Electrocardiographic analysis of each of the athletes revealed IRBBB in $10 \%$ (3 out of 30). First-degree AV block was diagnosed in 7\% (2 out of 30) of the athletes. No other abnormal findings were apparent in the ECG readings.

Echocardiography was performed on all athletes. Measurement findings are presented in Table 3 , including $\mathrm{Z}$-scores and literature values in athletes and nonathletes for comparison. All of our data fell within the reported values for athletes and nonathletes, ${ }^{15}$ except for the diameter of the left atrium. With regard to the left atrial diameter, our values were much smaller. The anatomic findings were limited to $1^{\circ}$ tricuspid insufficiency in 10 , mitral valve prolapse in 1 , and $1^{\circ}$ pulmonary insufficiency in 1 participant.

Fourteen climbers received echocardiographic examinations on 2 occasions (Table 4). The Z-scores for IVSD and LVPW had increased significantly over the timeframe of 27.5 mo, but the measurements for LVEDD did not change significantly. There was no change over time in the E/A ratio in the climbers or the group of Nordic skiers.

Although the measurements for the left ventricular dimension were significantly higher in the group of Nordic skiers at the time of the first examination, by the time of the second examination, the measurements for IVSD and LVPW had become comparable: They had significantly increased in the group of climbers but not in the group of skiers. Only LVEDD remained significantly lower in the group of climbers at the second examination (Figures 1 and 2).

Noteworthy findings during echocardiography included a single case of $1^{\circ}$ tricuspid insufficiency, $1^{\circ}$ pulmonary insufficiency, and mild mitral valve prolapse. All of these findings were still within physiologic limits and were not considered pathologic.

\section{Discussion}

The risk of SCD is twice as great for young athletes during sports participation than for nonathletes. ${ }^{28}$ Especially after

Table 3. Echocardiographic measurements of 12 girls and 18 boys from the GJNT (means and Z-score in parentheses) compared to literature findings of athletes

\begin{tabular}{llllll}
\hline Parameter & Boys & Literature boys & Girls & Literature girls & Literature nonathletes \\
\hline LVEDD (mm) & $48(-0.1)$ & $48^{15}$ & $44(-0.5)$ & $45^{15}$ & $43^{15}$ \\
IVSD $(\mathrm{mm})$ & $8(0)$ & $8^{15}$ & $8(0.3)$ & $8^{15}$ & $7^{15}$ \\
LVPW $(\mathrm{mm})$ & $8(0.5)$ & $8^{15}$ & $8(0)$ & $8^{15}$ & $7^{15}$ \\
E/A & 2 & $2^{15}$ & 2.1 & $2.0^{15}$ & $1.9^{15}$ \\
Left atrium $\left(\mathrm{cm}^{2}\right)$ & 15 & $24^{29}$ & 14 & $20^{29}$ & \\
\hline
\end{tabular}

E/A, ratio of early to late ventricular filling velocities; IVSD, interventricular septum thickness during diastole; LVEDD, left ventricular end-diastolic diameter; LVPW, left ventricular posterior wall thickness 
Table 4. Echocardiographic measurements (mean \pm SD in brackets) of the 8 girls and 6 boys from the German junior climbing team and junior national team of Nordic skiing at first and second examination

\begin{tabular}{lllll}
\hline \multirow{2}{*}{ Measurements } & First visit & & \multicolumn{2}{l}{ Second visit } \\
\cline { 2 - 4 } & Climber & Nordic skier & Climber & Nordic skier \\
\hline Z-score LVEDD & $-0.3 \pm 1.1$ & $0.7 \pm 0.8$ & $-0.2 \pm 1.2$ & $1.1 \pm 1.0$ \\
Z-score IVSD & $-0.1 \pm 1.3$ & $1.6 \pm 1.0$ & $0.8 \pm 0.8$ & $1.0 \pm 0.9$ \\
Z-score LVPW & $-0.1 \pm 1.0$ & $1.0 \pm 1.1$ & $0.5 \pm 0.6$ & $0.6 \pm 0.6$ \\
E/A & $2.2 \pm 0.8$ & $2.7 \pm 1.0$ & $2.3 \pm 0.5$ & $2.6 \pm 0.8$ \\
\hline
\end{tabular}

E/A, ratio of early to late ventricular filling velocities; IVSD, interventricular septum thickness during diastole; LVEDD, left ventricular end-diastolic diameter; LVPW, left ventricular posterior wall thickness

the first reported SCD in a young Canadian sports climber during a competition in the fall of 2019 (cause yet unknown), the importance for screening young elite sport climbers has risen. So far there is no data about the sports-specific cardiac adaptations to be expected in elite climbers. The purpose of this study was to use existing ECG and echocardiography data from the yearly medical examination of the GJNTC to get a first impression of the possible effects of climbing on the left ventricular measurements of elite climbers.

Pediatric athletes are known to have a longer PR interval (148 vs $139 \mathrm{~min}$ ) and a significantly greater frequency of sinus bradycardia (46\%), first-degree AV block (2 vs $<1 \%$ ), IRBBB (26 vs $8 \%$ ), voltage criteria for LVH (35 vs $24 \%$ ), and early repolarization (37 vs $29 \%$ ) when

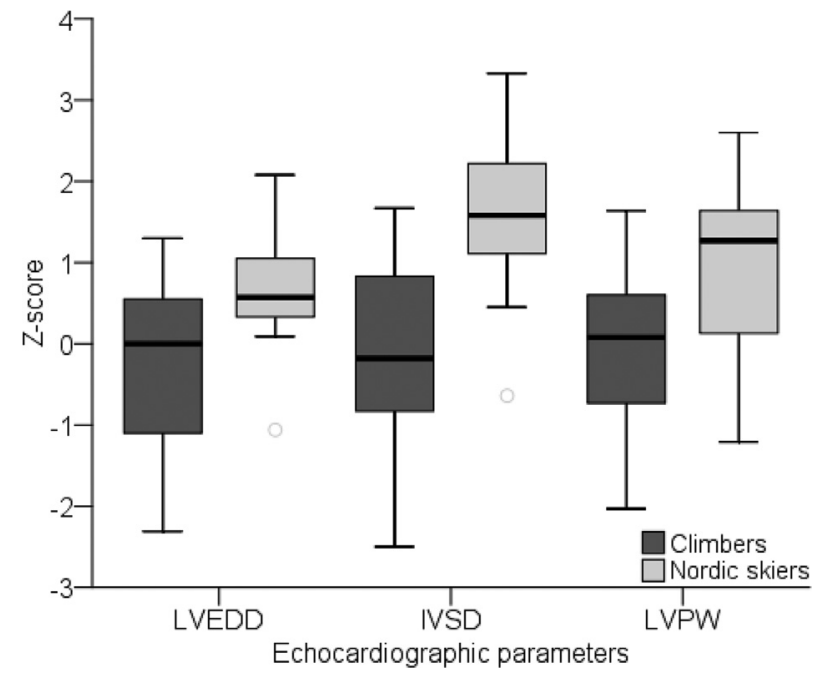

Figure 1. Z-scores of left ventricular end-diastolic diameter, interventricular septum thickness during diastole, and left ventricular posterior wall thickness of climbers and Nordic skiers at the first visit, depicted as boxplots elements. *Significant difference between the 2 groups. compared with pediatric nonathletes. ${ }^{15}$ In our study, the frequency of first-degree AV block and IRBBB was comparable to these reported observations. Bradycardia, LVH, and early repolarization were not found. Thus, the adaptations in our collection of elite climbers are comparable to those seen in other sports. There were no pathologic findings.

The main morphologic feature of the athlete's heart in adults is LVH, involving an increase in both the internal diameter and wall thickness. ${ }^{26}$ These athletic features are induced mostly by the effects of endurance training. ${ }^{17}$ The structural adaptations of the pediatric athlete's heart are presented in a systematic review and meta-analysis. ${ }^{15}$ The authors found that pediatric athletes had a significantly greater LVEDD, IVSD, LVPW, and left atrial diameter

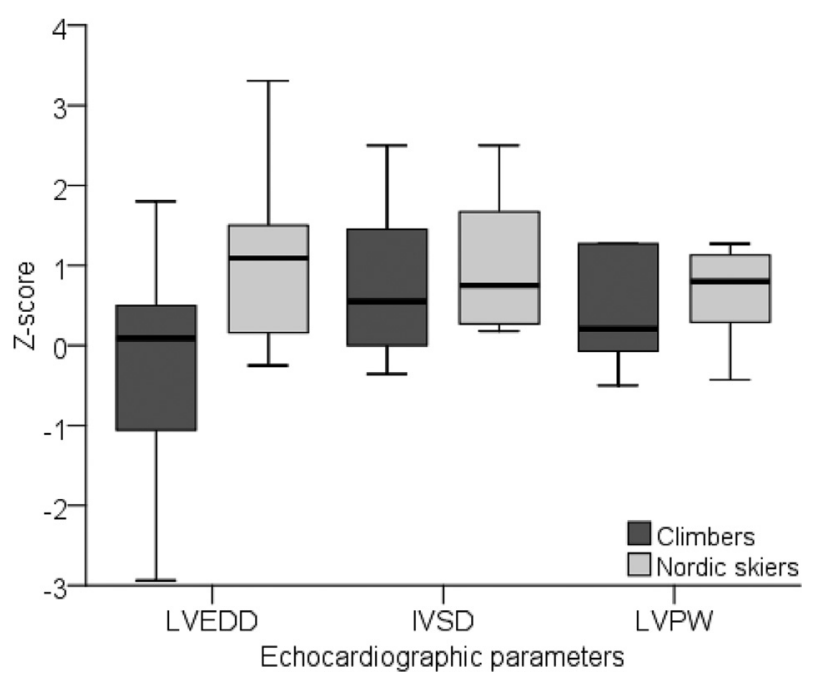

Figure 2. Z-scores of left ventricular end-diastolic diameter, interventricular septum thickness during diastole, and left ventricular posterior wall thickness of climbers and Nordic skiers at the second visit after $2 \mathrm{y}$, depicted as boxplots elements. * Significant difference between the 2 groups. 
than nonathletes. Our data fell between the values recorded for athletes and those for nonathletes.

It has also been suggested that Z-scores should be used to account for somatic growth. Whereas in adult cardiology, echocardiographic measurements can be reported with respect to normal range, this is impossible in children because the normal range of measurements is affected by patient growth and/or age. ${ }^{29}$ As a description of clinical and echocardiographic variables in children, the measurements are therefore often reported as Z-scores. Standard scores indicate how many standard deviations the measurements lie above or below a size- and age-specific population. ${ }^{29}$ When analyzing the Z-scores of the GJNTC, they all fall within normal limits. However, 2 y of regular physical training are needed to develop characteristics of the athlete's heart. ${ }^{25}$ Interestingly, the longitudinal data of our climbers revealed that all Z-score of the left ventricle (LV) increased over a timeframe of $27.5 \mathrm{mo}$, but only the measurements of IVSD changed significantly. Furthermore, even though the left ventricular dimensions were significantly lower when compared to elite Nordic skiers at an age of $16 \mathrm{y}$, they were not significantly different with respect to the thickness of the interventricular septum (IVSD) or the lateral wall (LVPW) of the LV when evaluated again after $27.5 \mathrm{mo}$.

In Nordic skiing, the average speed achieved by the athlete depends mainly on the metabolic energy turnover maintained by the athlete during the workout divided by the economy of progression, or energy cost of locomotion. ${ }^{30,31}$ Thus, Nordic skiing is generally classified as an endurance or dynamic sport. Dynamic sports are characterized by involving a high amount of movement and only minimal amount of force. This leads to increases in heart rate and stroke volume (cardiac output). Dynamic sports thus have a high volume load on the heart. As a consequence, enlargement of the left ventricular internal diameter and a proportional increase in wall thickness can be expected, termed eccentric LVH. ${ }^{32}$ Static or so-called resistance sports, such as weightlifting, martial arts, and field throwing, involve minimal movement but a high amount of force. ${ }^{33}$ This in turn leads to an elevation of cardiac output as a consequence of an increase in heart rate and blood pressure, causing pressure load on the heart. The so-called concentric LVH that results from static sports includes thickening of the ventricular wall with unchanged internal dimension. ${ }^{32}$ Nordic skiing is a pure endurance sport, and thus all the signs of eccentric LVH can be expected. However, climbing probably falls more into the category of team or combat sports, ${ }^{9}$ and it is unclear what kind of adaptation, if any at all, can be expected.

The finding that the wall thickness of the LV reached measurements comparable to those of the Nordic skiers after being on the team for $2 \mathrm{y}$ was surprising. The fact that the internal diameter of the LV did not change significantly over time, nor did it reach dimensions comparable to the diameter of the LV of the Nordic skiers, suggests eccentric hypertrophy. As a consequence of the small number of athletes investigated cross-sectionally in this study, a classification of climbing as a high-static sport should be discouraged at this time. Interestingly, there were no significant adaptations in the LV of the Nordic skiers after 28.7 mo. A possible explanation for these findings could be that the climbers entered the GJNTC at an early stage in their climbing career. Up to this point, most of them had not undertaken a sport-specific training regimen. Although climbing has been accepted as an Olympic sport, pronounced differences in the structure and organization of training methods exist, in contrast Nordic skiing, which is a well-developed sport with large training facilities and an early talent recruitment system in Germany. It can be expected that the Nordic skiers had already been subjected to intensive training before their first echocardiographic examination. Therefore, their cardiac adaptations were less pronounced over the examined timeframe. In contrast, the climbers who entered the GJNTC may have only started a regular training regimen; this may explain the cardiac adaptations observed in our study. Future prospective studies should take historic training diaries into account. However, because we did not record their training regimen before or after entering the team, this is only a hypothesis.

Although no pathologic findings were recorded during the echocardiographic examination, special attention needs to be paid to the anatomic evaluation of the young athlete's heart because most of them will never have undergone echocardiography. If anatomic findings are revealed, further investigations are warranted and cardiopulmonary exercise testing should be discouraged until the results have been discussed with a pediatric cardiologist.

\section{LIMITATIONS}

Our study had several limitations. First, the design of the study is retrospective in nature, which is a limitation in and of itself. The echocardiographic data did not follow a specific protocol available in the literature. ${ }^{27}$ This is because the data were obtained from the yearly medical examination of the GJNTC and primarily not designed as a prospective study. Future studies need to evaluate more parameters following literature protocols in a prospective manner to achieve a clearer picture of the cardiac adaptations of elite climbers. Furthermore, the sample is rather heterogeneous and presents young athletes over a large age span. This is because study entry was defined by acceptance to the GJNTC, which is solely based on climbing ability. For the longitudinal design, the number of athletes is comparably small because of high turnover of athletes 
within the team. Furthermore, there was no age-matched control group of sedentary or nonelite subjects, which is why literature data and Z-scores were used for comparison.

\section{Conclusions}

Our findings suggest that the young elite athletes of the GJNTC underwent cardiac adaptations in terms of LV wall thickness comparable to elite Nordic skiers. The internal LV diameter did not change and remained significantly smaller than that of the Nordic skiers, which may be explained by eccentric hypertrophy, as observed in static sports, rather than concentric hypertrophy, as observed in dynamic sports. However, we urge caution because the number of participants was small. More important to note is that all findings were within normal limits and no team member showed signs of cardiomyopathy or congenital heart disease. Further longitudinal and prospective studies are needed to more fully evaluate the long-term effects of climbing on the heart of the athlete.

Author Contributions: Conception and design and analysis and interpretation of data; drafting the article; and final approval of the version to be published (IS). Conception and design and analysis and interpretation of data; revising the article critically for important intellectual content; and final approval of the version to be published (JW). Drafting and revising the article critically for important intellectual content; final approval of the version to be published (GJ). Analysis and interpretation of data; revising the article critically for important intellectual content; and final approval of the version to be published (SD). Conception and design and analysis and preparation of data interpretation of data; revising the article critically for important intellectual content; and final approval of the version to be published (IS, JW, GJ, SD, CL, VS).

Financial/Material Support: None. Disclosures: None.

\section{References}

1. Baumgartner L, Schulz T, Oberhoffer R, Weberruß H. Influence of vigorous physical activity on structure and function of the cardiovascular system in young athletes-The MuCAYA-Study. Front Cardiovasc Med. 2019;6:148.

2. Sharma S, Merghani A, Mont L. Exercise and the heart: the good, the bad, and the ugly. Eur Heart J. 2015;36(23): 1445-53.

3. Corrado D, Basso C, Schiavon M, Thiene G. Does sports activity enhance the risk of sudden cardiac death? J Cardiovasc Med (Hagerstown). 2006;7(4):228-33.

4. Sharma S. Athlete's heart-effect of age, sex, ethnicity and sporting discipline. Exp Physiol. 2003;88(5):665-9.

5. Chandra N, Papadakis M, Sharma S. Preparticipation screening of young competitive athletes for cardiovascular disorders. Phys Sportsmed. 2010;38(1):54-63.

6. Maron BJ. Hypertrophic cardiomyopathy: a systematic review. JAMA. 2002;287(10):1308-20.

7. Maron BJ, Gardin JM, Flack JM, Gidding SS, Kurosaki TT, Bild DE. Prevalence of hypertrophic cardiomyopathy in a general population of young adults. Echocardiographic analysis of 4111 subjects in the CARDIA Study. Coronary artery risk development in (young) adults. Circulation. 1995;92(4):785-9.

8. Chandra N, Bastiaenen R, Papadakis M, Sharma S. Sudden cardiac death in young athletes: practical challenges and diagnostic dilemmas. J Am Coll Cardiol. 2013;61(10):1027-40.

9. Schöffl I, Wustenfeld J, Jones G, Dittrich S, Lutter C, Schöffl V. Cardiopulmonary exercise tests of adolescent elite sport climbers-a comparison of the German junior national team in sport climbing and Nordic skiing. Wilderness and Environmental Medicine. 2020 [Epub ahead of print].

10. Corrado D, Basso C, Pavei A, Michieli P, Schiavon M, Thiene G. Trends in sudden cardiovascular death in young competitive athletes after implementation of a preparticipation screening program. JAMA. 2006;296(13): 1593-601.

11. Drezner JA, Rao AL, Heistand J, Bloomingdale MK, Harmon KG. Effectiveness of emergency response planning for sudden cardiac arrest in United States high schools with automated external defibrillators. Circulation. 2009;120(6): 518-25.

12. Harmon KG, Asif IM, Klossner D, Drezner JA. Incidence of sudden cardiac death in National Collegiate Athletic Association athletes. Circulation. 2011;123(15):1594-600.

13. Winkel BG, Risgaard B, Bjune T, Jabbari R, Lynge TH, Glinge $\mathrm{C}$, et al. Gender differences in sudden cardiac death in the young-a nationwide study. BMC Cardiovasc Disord. 2017;17(1):19.

14. De Innocentiis C, Ricci F, Khanji MY, Aung N, Tana C, Verrengia E, et al. Athlete's heart: diagnostic challenges and future perspectives. Sports Med. 2018;48(11):2463-77.

15. McClean G, Riding NR, Ardern CL, Farooq A, Pieles GE, Watt V, et al. Electrical and structural adaptations of the paediatric athlete's heart: a systematic review with meta-analysis. Br J Sports Med. 2018;52(4):230.

16. Landry $\mathrm{CH}$, Allan KS, Connelly KA, Cunningham K, Morrison LJ, Dorian P, et al. Sudden cardiac arrest during participation in competitive sports. N Engl J Med. 2017;377(20): 1943-53.

17. Maisch B. Exercise and sports in cardiac patients and athletes at risk: balance between benefit and harm. Herz. 2015;40(3): 395-401.

18. Corrado D, Pelliccia A, Bjornstad HH, Vanhees L, Biffi A, Borjesson M, et al. Cardiovascular pre-participation screening of young competitive athletes for prevention of sudden death: proposal for a common European protocol. Consensus Statement of the Study Group of Sport Cardiology of the Working Group of Cardiac Rehabilitation and Exercise Physiology and the Working Group of Myocardial and Pericardial Diseases of the European Society of Cardiology. Eur Heart J. 2005;26(5): 516-24.

19. Grazioli G, Merino B, Montserrat S, Vidal B, Azqueta M, Pare C, et al. Usefulness of echocardiography in preparticipation screening of competitive athletes. Rev Esp Cardiol (Engl Ed). 2014;67(9):701-5.

20. Yim ES, Basilico F, Corrado G. Early screening for cardiovascular abnormalities with preparticipation echocardiography: utility of focused physician-operated echocardiography 
in preparticipation screening of athletes. J Ultrasound Med. 2014;33(2):307-13.

21. Gilbert CA, Nutter DO, Felner JM, Perkins JV, Heymsfield SB, Schlant RC. Echocardiographic study of cardiac dimensions and function in the endurance-trained athlete. Am J Cardiol. 1977;40(4):528-33.

22. Morganroth J, Maron BJ, Henry WL, Epstein SE. Comparative left ventricular dimensions in trained athletes. Ann Intern Med. 1975;82(4):521-4.

23. Pelliccia A, Maron BJ, Spataro A, Proschan MA, Spirito P. The upper limit of physiologic cardiac hypertrophy in highly trained elite athletes. N Engl J Med. 1991;324(5):295-301.

24. Galderisi M, Cardim N, D'Andrea A, Bruder O, Cosyns B, Davin L, et al. The multi-modality cardiac imaging approach to the athlete's heart: an expert consensus of the European Association of Cardiovascular Imaging. Eur Heart J Cardiovasc Imaging. 2015;16(4):353.

25. Pavlik G, Major Z, Csajagi E, Jeserich M, Kneffel Z. The athlete's heart. Part II: influencing factors on the athlete's heart: types of sports and age (review). Acta Physiol Hung. 2013;100(1):1-27.
26. Pavlik G, Major Z, Varga-Pinter B, Jeserich M, Kneffel Z. The athlete's heart Part I (Review). Acta Physiol Hung. 2010;97(4):337-53.

27. Grazioli G, Sanz M, Montserrat S, Vidal B, Sitges M. Echocardiography in the evaluation of athletes. F1000Res. 2015;4:151.

28. Corrado D, Basso C, Rizzoli G, Schiavon M, Thiene G. Does sports activity enhance the risk of sudden death in adolescents and young adults? J Am Coll Cardiol. 2003:42(11):1959-63.

29. Chubb H, Simpson JM. The use of Z-scores in paediatric cardiology. Ann Pediatr Cardiol. 2012;5(2):179-84.

30. di Prampero PE. Factors limiting maximal performance in humans. Eur J Appl Physiol. 2003;90(3-4):420-9.

31. di Prampero PE, Atchou G, Brückner JC, Moia C. The energetics of endurance running. Eur J Appl Physiol Occup Physiol. 1986;55(3):259-66.

32. Fagard R. Athlete's heart. Heart. 2003;89(12):1455-61.

33. Barbier J, Ville N, Kervio G, Walther G, Carre F. Sports-specific features of athlete's heart and their relation to echocardiographic parameters. Herz. 2006;31(6):531-43. 